\title{
The Social Content Strategy of the ATLAS Collaboration
}

\author{
Antonio Sidoti* \\ on behalf of the ATLAS Collaboration. \\ Istituto Nazionale Fisica Nucleare - Sezione di Bologna \\ E-mail: antonio.sidoti@bo.infn.it
}

Social media is an essential tool for communicating particle-physics results to a wide audience. This presentation will explore how the nature of social media platforms has impacted the content being shared across them, and the subsequent effect this has on the user experience. The ATLAS Experiment at the LHC has adapted its communication strategy to match this social media evolution, producing content specifically targeting this emerging audience. The success of this approach is examined and the effect on user experience is evaluated.

Sixth Annual Conference on Large Hadron Collider Physics (LHCP2018)

4-9 June 2018

Bologna, Italy

${ }^{*}$ Speaker. 


\section{Introduction}

Science outreach using social media is a relatively young activity in fast development. Few of today's social media platforms existed ten years ago and the field is in constant evolution. Therefore the potential to perform science outreach is huge, but the competition on the social networks is large. The strategy for science outreach has to adapt to the rapidly changing environment, trends, technological platforms, media etc.

The ATLAS experiment at LHC [1] employs various social media platforms [2] to communicate their research and achievements to a wider public audience. This audience is composed of scientists of other fields (i.e. not directly involved in particle-physics), students at different stages (elementary/primary school to first-year university students), general public and policy makers. Each group has different reasons to be engaged which include: be informed and learn, be aware of science as a peculiar and inclusive social model, bring future scientists to particle-physics, justify public funding, and, last but not least, share the beauty of fundamental research.

In these proceedings we will review how the social media strategy of the ATLAS collaboration has changed recently to keep pace with the evolution of technology and social platforms by modifying the format of the messages.

\section{Social Media}

The majority of U.S. adults now use social media at least once per month. The most popular social platform in 2018 is YouTube (73\%) followed by Facebook (68\%) and Instagram. Instagram registered the largest increase from 2012 passing from 10\% to 35\% [3] (Fig. 1 left).

At the same time, the popularity of many social media platforms varies according to the age of the users. While Facebook seems to be used almost uniformly in the U.S. by different age groups (around $80 \%$ for U.S. adults in the 18 to 49 age interval), Instagram is more likely to be used by younger U.S. adults ( $71 \%$ for the 18 to 24 years compared to $40 \%$ and $21 \%$ for the $[30,49]$ and [50,64] age interval respectively) [3] (Fig. 1 right). Therefore to ensure an optimal coverage of different age intervals it is important to efficiently use various social media.

Since 2015, mobile devices (including both smartphones and tablets) have become the primary means of accessing social media for most users, accounting, overall, for $73 \%$ of internet consumption in 2018. This is up from 70\% in 2017 and 65\% in 2016. The fraction of mobile access is even larger for social media users from developing countries than developed ones, giving a great opportunity to close the developed versus developing countries gap at least for accessing material published on social media. For example, exclusive access from mobile is about four times larger in India with respect to accessing from desktop [4].

Mobile dominance imposes new standards on social media strategy: for example mobile users are less likely to open webpages, preferring to remain in-app when viewing new content. Concisely: You can lead a user to contents, but you can't make them click!

Adapting ATLAS outreach to this new means of consumption is vital for ensuring that ATLAS content reaches its target audiences. 

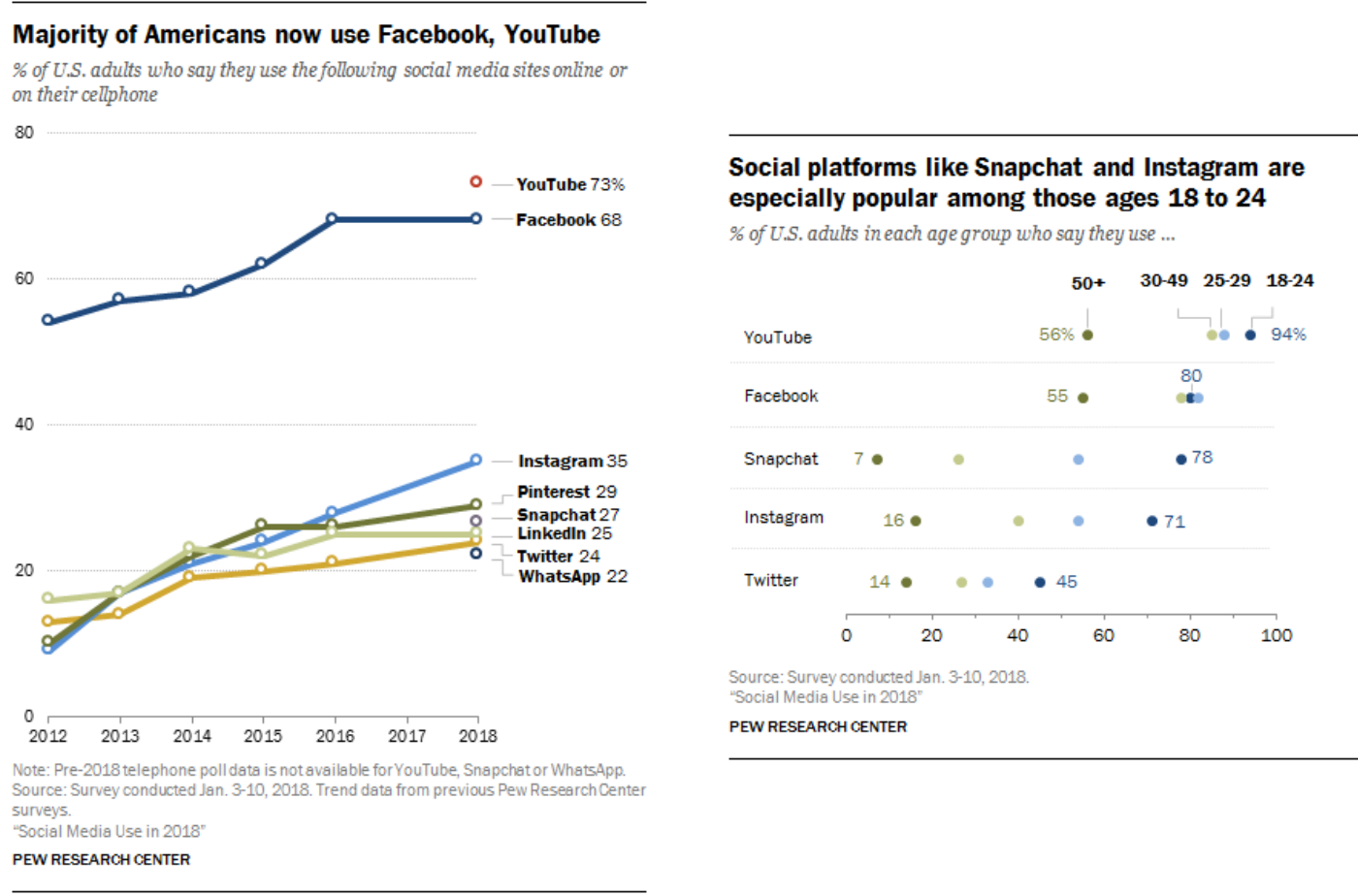

Figure 1: Left: Use of different social media platforms by various age groups. Right: Fraction of U.S. adults who say they use the following social media sites online or on their cellphone. [3].

\section{ATLAS Strategy}

ATLAS is therefore tailoring its messages to each social media platform, addressing the benefits and limitations of each, focusing on making ATLAS content more accessible to mobile users, thus improving its visibility and share-ability.

In particular, Facebook posts with more extensive scientific contents (like physics briefings, press statements) have been replaced by Facebook notes allowing access directly through Facebook, instead just of linking to the article on the ATLAS or CERN webpage. The entire content is available without clicking away from Facebook. ATLAS Event Displays are attractive images to use as Facebook notes require banners.

It has been measured that Facebook notes perform consistently better than links to the ATLAS website, with an average of $\sim 145$ readers compared to $\sim 90$ link clicks [5].

Social media videos tend to be more direct and capture attention quickly. Therefore it is important to tailor them to the various platforms. Videos with traditional $16 \times 9$ aspect ratio are ideal playing with sound on non-mobile platforms like laptops or desktops. The optimal duration of these movies is around 4 minutes. They tend to be published using traditional platforms like YouTube or on the CERN Document Server (CDS). They also adopt a more traditional movie narrative.

On the contrary, videos published on Twitter, Instagram and Facebook use a different format and narrative. Firstly, square videos (aspect ratio $1 \times 1$ ) appear larger on mobile screens than those with standard $16 \times 9$ ratio.

Twitter and Instagram have one and two minutes duration limit respectively, therefore, most 
of videos produced and published on Facebook, Twitter and Instagram are now about one minute long. Four out of five Facebook users watch videos on Facebook and Instagram without sound, therefore videos now have subtitles and captions. This also eases the access to video content for non-English native speakers since reading text is simpler than listening to audio content (Figure 2). This also helps impared hearing people, increasing thus the content accessibility.

Recently, the ATLAS collaboration adapted the video it produces to the $1 \times 1$ aspect ratio, limiting the length to one minute as above and adding captions or subtitles. Also not limiting content exclusively to physics results, but extending them to the real life of researchers (picturing for example diversity [6]) can increase the popularity of ATLAS contents on social media. The "Women in Physics" social media video for International Woman's Day (IWD2018) [7] has reached 49k views on Facebook, 13k views on Twitter and 1,300 views on Instagram, making it the most popular ATLAS video published since the start-up of the LHC. On average, video sharing has more than doubled and despite shorter length, globally, more minutes are watched.
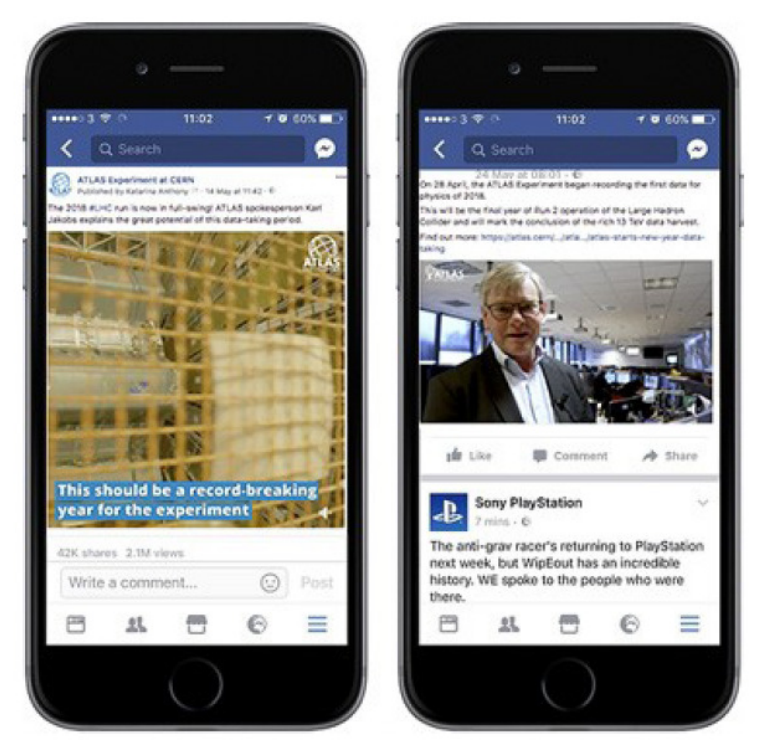

Figure 2: Mobile tailored (left) versus traditional (right) format of videos published by ATLAS on Facebook profile @ATLASexperiment.

\section{Conclusion}

The exponential increase of social media usage and mobile-only users led the ATLAS collaboration to adapt its social media strategy, modifying both video making and publishing of physics briefings leading to an increase of social media presence of ATLAS related contents. The amount of scientific information publicly available on the internet is rapidly increasing.

As scientists, we cannot enclose ourselves in our labs. We should ensure that we are consistently sharing, explaining, and providing solid justification for our results to provide a convincing narrative against anti-scientific prejudices and manipulations. 
If scientists do not venture outside our labs to talk about the scientific method and explain our research achievements to non-experts, we abandon this narrative to outsiders, allowing the public view of our work to be shaped by people who may not fully understand it.

\section{Acknowledgment}

On behalf of the ATLAS outreach and social media team, we wish to thank the whole ATLAS Collaboration for providing us with ideas and contents (i.e. physics results) to share with the world. Without excellent physics results you cannot have good communication!

\section{References}

[1] ATLAS Collaboration, "The ATLAS Experiment at the CERN Large Hadron Collider", JINST, 3 S08003, (2008)

[2] https://www. facebook.com/ATLASexperiment/

https://twitter.com/ATLASexperiment

https://www.instagram.com/atlasexperiment/

[3] Pew Research Centre, "Social Media Use in 2018", http://www. pewinternet.org/2018/03/01/social-media-use-in-2018/, (2018).

[4] comScore, "Global Digital Future in Focus", https : / /www. comscore.com/, (2018)

[5] Nellist, C., "Social Media Strategy for the ATLAS experiment", ATL-OREACH-PROC-2016-008, (2016).

[6] Biondi, S., "Picturing diversity in the ATLAS Experiment", POS (EPS-HEP2017), 553 (2017).

[7] International Woman's Day 2018 ATLAS Video

https://www. facebook.com/ATLASexperiment/videos/1793176540719767/ 